\title{
OTR IMAGING OF INTENSE 120 GEV PROTONS IN THE NUMI BEAMLINE AT FNAL *
}

\author{
V. E. Scarpine ${ }^{\#}$, G. R. Tassotto, FNAL, Batavia, IL 60542 U.S.A. \\ A. H. Lumpkin, ANL-APS, Argonne, IL 60439, U.S.A.
}

\begin{abstract}
An Optical Transition Radiation (OTR) detector has been installed in the Fermilab NuMI proton beamline, which operates at beam powers of up to $\sim 350 \mathrm{~kW}$, to obtain real-time, spill-by-spill beam profiles for neutrino production. As part of the Run II collider plan and the NuMI neutrino program, a series of OTR detectors were designed, constructed and installed in various beamlines at Fermilab. NuMI OTR images of $120 \mathrm{GeV}$ protons for beam intensities up to $4.1 \times 10^{13}$ at a spill rate of $0.5 \mathrm{~Hz}$ and small transverse beam size of $\sim 1 \mathrm{~mm}$ (sigma) are presented here. Beam profiles are extracted from the OTR images and compared with an adjacent secondaryelectron emission (SEM) monitor. The OTR detector provides two-dimensional beam shape, such as ellipticity and tilt, as well as beam centroid and beam intensity information. In addition, the response of the OTR detector over time is examined to look for signs of foil aging.
\end{abstract}

\section{INTRODUCTION}

Optical Transition Radiation (OTR) occurs when a charged particle transverses a material boundary where there is a change in the index of refraction. OTR is emitted in both the forward and the backward directions relative to the particle motion. The radiation pattern is a radially polarized cone of light with its peak intensity at an angle of $1 / \gamma$, where $\gamma$ is the particle Lorentz factor. The first observation of OTR was by Goldsmith and Jelley [1].

OTR detectors have been used extensively to measure transverse beam shape in electron accelerators. Recently, CERN incorporated OTR detectors in various transfer lines to measure proton bunches used for operation of the LHC [2]. As part of the Run II collider plan and the NuMI neutrino program, a series of OTR detectors were designed, constructed and installed in various beamlines at Fermilab. Previous near-field OTR images, from other Fermilab beamlines, of lower-intensity $120 \mathrm{GeV}$ and 150 $\mathrm{GeV}$ protons with larger transverse beam size have been presented [3] [4] [5].

\section{EXPERIMENTAL SETUP}

\section{OTR Detector for NuMI}

A collaboration of Fermilab and Argonne National Laboratory developed an OTR detector design optimized to measure both proton and antiprotons in the energy range of 120 to $150 \mathrm{GeV}$ and for bunch intensities from

\footnotetext{
* Work supported by U. S. Department of Energy under Contract No. DE-AC02-CH03000 and by the U. S. Department of Energy, Office of Basic Energy Sciences, under Contract No. DE-AC02-06CH11357. \# scarpine@fnal.gov, tassotto@fnal.gov, lumpkin@aps.anl.gov
}

$\sim 5 \mathrm{e} 10$ to $\sim 1 \mathrm{e} 14$ particles. Figure 1 shows a block diagram of these detectors. The NuMI OTR detector uses two independently inserted 6 micron Kapton foils with 0.12 micron of aluminum. These foils reduce the proton beam scatter by $70 \%$ compared with an adjacent SEM monitor (SEM). The foils are tilted 45 degree to the beam axis in order to reflect the backward OTR perpendicular to the beamline. One foil is used as a primary OTR screen while the second foil is periodically inserted into the beam to track changes to the primary foil.

In addition, the detector utilizes a radiation-hardened CID camera [6] that can be rotated to the Scheimpflug angle [7] to maintain focus across the foil. The detector also has two filter wheels consisting of neutral density filters and two orthogonal polarizing filters. The focus lens can switch between near-field and far-field imaging.

The detector acquires OTR images for every beam pulse. A front-end PC uses a Labview acquisition and analysis program to process these images. The analysis produces the beam $\mathrm{X}$ and $\mathrm{Y}$ centroids and sigmas as well as the beam tilt and ellipticity. A more detailed description of the OTR detector can be found in reference [8].

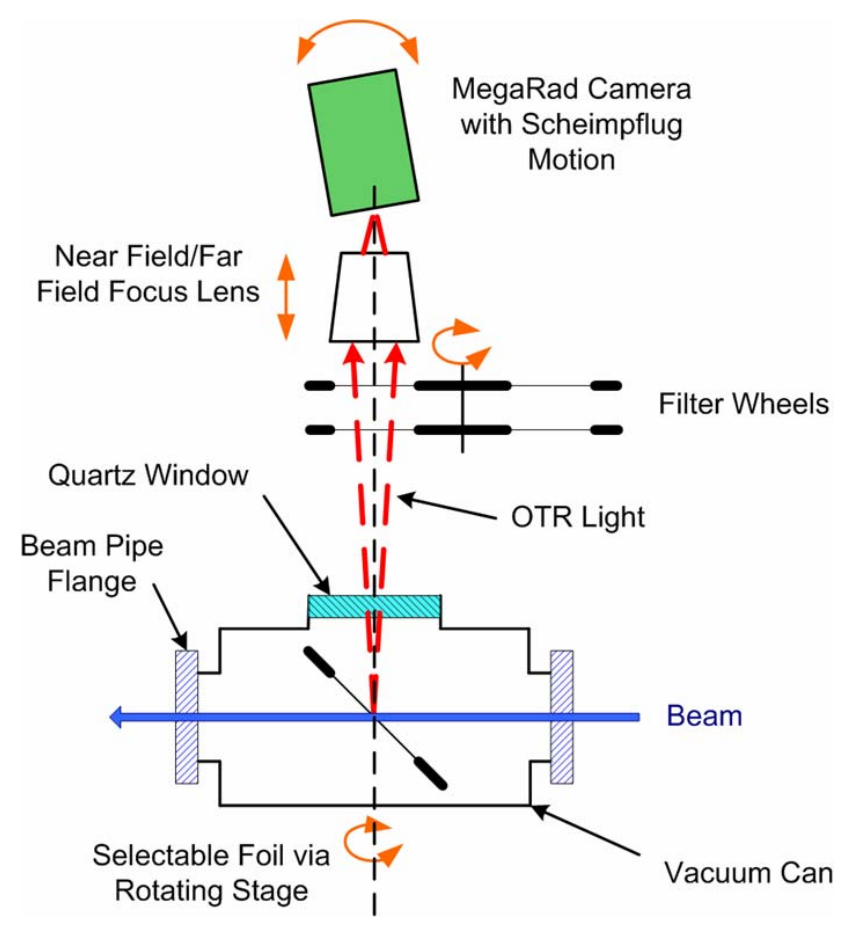

Figure 1: Block diagram of FNAL OTR detector.

\section{NuMI Beamline}

The Neutrinos at the Main Injector (NuMI) beamline is used to generate intense $v_{\mu}$ beams via the decay of pion and kaon secondaries produced from $120 \mathrm{GeV}$ protons 
impacting the NuMI target [9]. The beamline was initially designed to accept up to $4 \times 10^{13} 120 \mathrm{GeV}$ protons per pulse at a repetition rate of 0.53 hertz. Recent Main Injector studies have supplied $4.1 \times 10^{13}$ protons per pulse at 0.45 hertz to the NuMI target. Normal operation of the NuMI beamline switches between two modes. During most operating periods NuMI receives $\sim 2.4 \times 10^{13}$ protons per pulse at the target. During short periods where there is no antiproton production, NuMI receives $\sim 3.2 \times 10^{13}$ protons per pulse from the Main Injector.

Presently, NuMI uses a segmented titanium foil SEM just upstream of the NuMI target and shield wall as a target beam profile monitor [10]. This target SEM measures the beam profile for every pulse. The NuMI OTR detector is installed just downstream of the target SEM but still in front of the shield wall.

\section{NUMI OTR MEASUREMENTS}

The NuMI OTR detector has taken measurements at various times over the past year. In March of 2007, the primary OTR foil was inserted into the NuMI beamline for continuous operation. Both near-field and far-field OTR images have been taken for beam intensities from $\sim 2.2 \times 10^{13}$ to $4.1 \times 10^{13}$. Far-field imaging will not be presented in this paper but is discussed in another paper presented at this conference [11]. Figure 2 shows a histogram of the beam pulse intensities during $\sim 80$ days of continuous operation. This corresponds to $\sim 6.5 \times 10^{19}$ protons through the primary OTR foil.

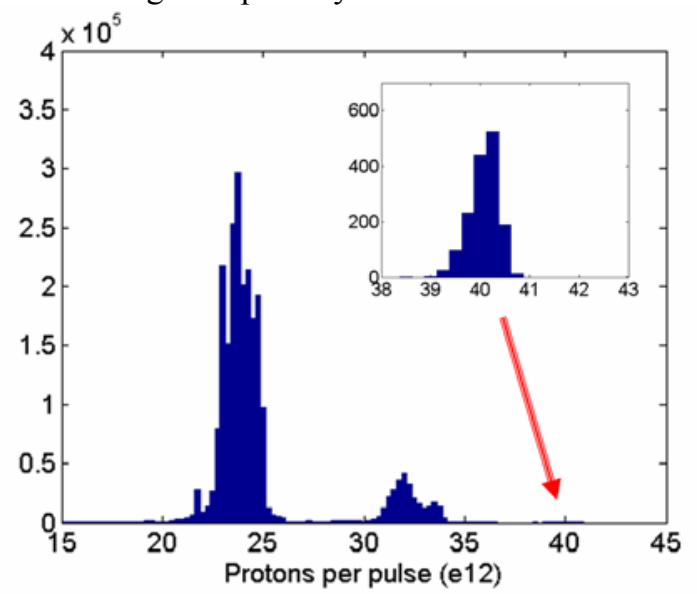

Figure 2: NuMI beam pulse intensities for $\sim 80$ days of operation while OTR foil is in beam path.

Figure 3 shows OTR images for bunch intensities of $\sim 2.4 \times 10^{13}$ and $\sim 4.1 \times 10^{13}$. This figure also shows the beam projections with Gaussian fits. The images show the increase in beam size with increase in beam intensity. The images also show an increase in the beam ellipticity with higher intensities. This shows the advantage of a twodimensional beam shape monitor, such as an OTR detector, over other standard one-dimensional profile monitors.
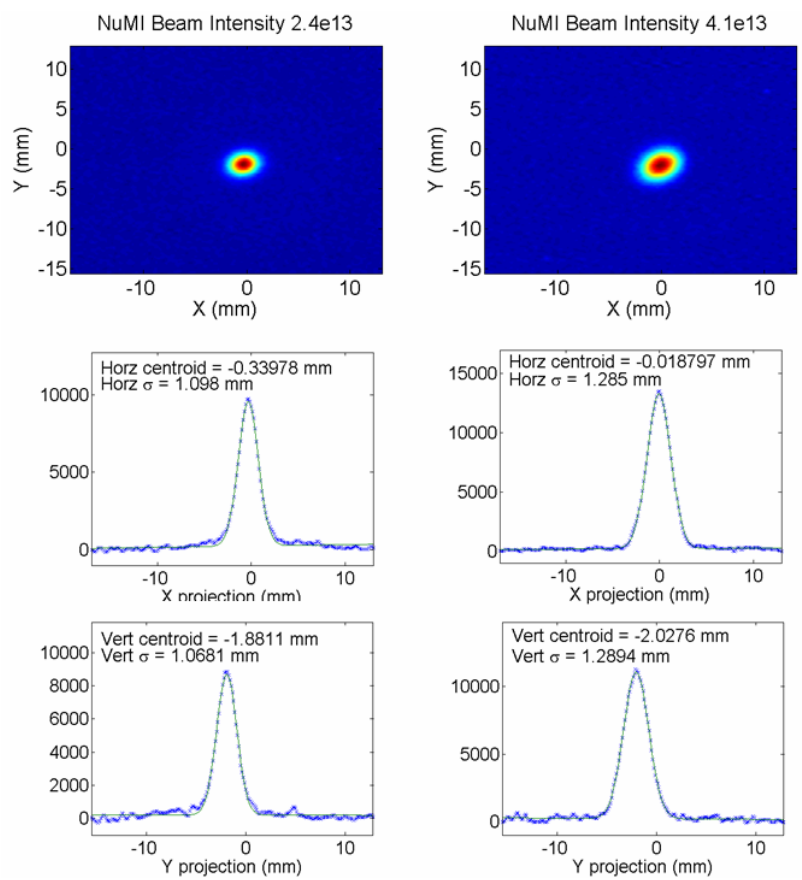

Figure 3: NuMI OTR beam images and horizontal and vertical projections with Gaussian fits for $2.4 \times 10^{13}$ and $4.1 \times 10^{13}$ protons per bunch.

\section{OTR Detector Response over Time}

The choice of an aluminized Kapton foil for the NuMI OTR was made to minimize the scatter of the NuMI beam. Issues of foil lifetime at this high beam intensity are not well understood but it was assumed that the foil would change over time. Figure 4 shows a comparison of the measured horizontal beam $\sigma$ from the SEM monitor and the OTR detector over a period of 80 days. One can see from the figure that value of $\sigma$ for the OTR detector is slowly diverging from the value from the SEM monitor. Although there is a difference in these two measurements, it is not clear that this could be attributed to a change in the OTR foil.

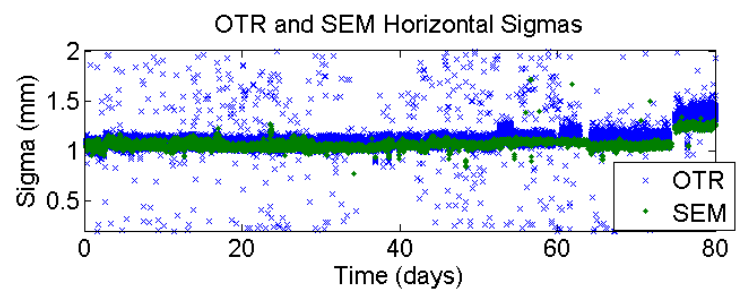

Difference in Horizontal Sigmas

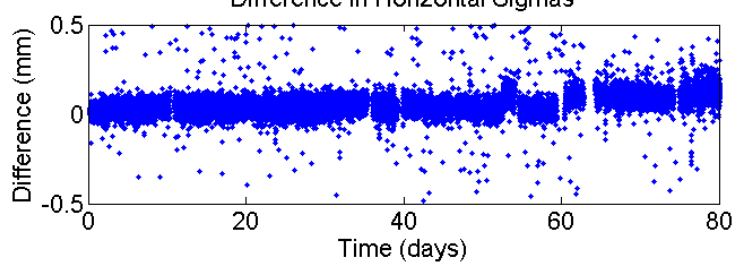

Figure 4: Comparison of the horizontal $\sigma$ between the SEM monitor and the OTR detector over time.

After $\sim 70$ days with the primary foil in the NuMI beamline, the secondary foil was temporarily inserted into 
the beamline. The OTR images from this foil were compared to OTR images taken with the primary foil to look for signs of change in the primary foil. Figure 5 shows OTR images and projections for the primary and secondary foils under similar beam conditions. These images give similar centroid and $\sigma$ values for both foils but the OTR intensity from the primary foil is clearly reduced.
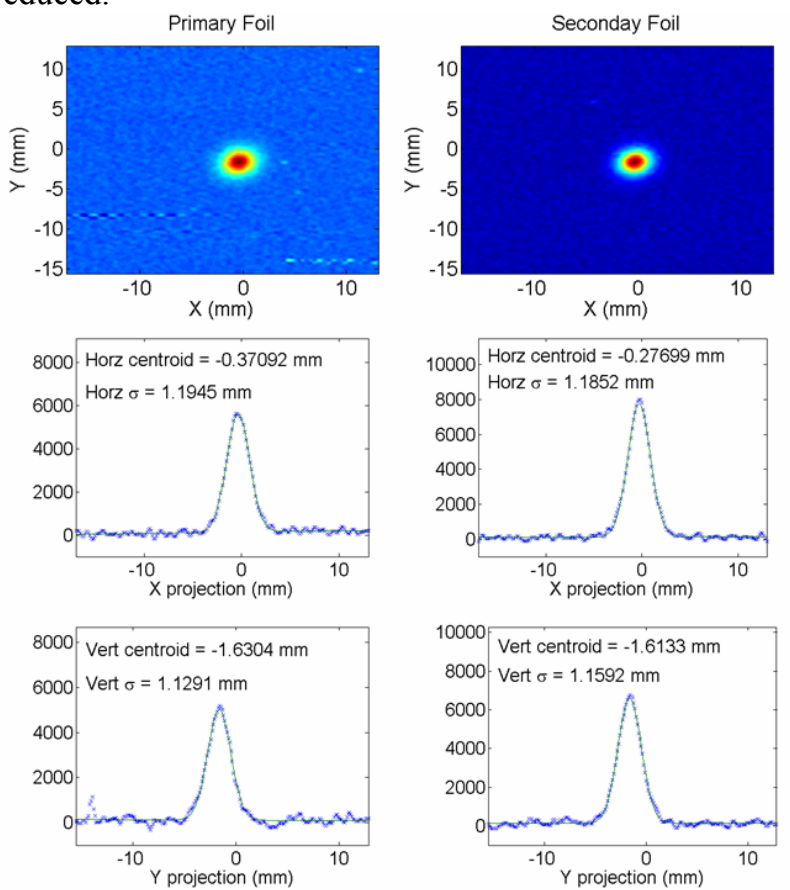

Figure 5: Comparison of OTR images taken with the primary and the secondary foils under similar beam conditions after $\sim 70$ days of primary foil operation.

\section{CONCLUSION}

We have successful operated an OTR detector upstream of the NuMI target with over $6.5 \times 10^{19} 120 \mathrm{GeV}$ protons. The generated OTR images give a good measure of the NuMI beam shape and these values compare well to the present SEM monitor. Early indications are that the OTR Kapton foil has aged but still produces acceptable images for beam shape measurements.

\section{ACKNOWLEDGEMENTS}

We thank our NuMI colleagues for their continued help and interest in this detector: Sam Childress, Peter Lucas, Gordon Koizumi, Jim Hylen and Doug Jenson. We also would like to thank the FNAL Accelerator Division for their contributions: Dave Slimmer, Dan Schoo, Rick Pierce, Linda Purcell-Taylor, Terry Anderson, Brad Tennis and Jim Fitzgerald. In addition, we wish to thank Carl Lindenmeyer, Jerry Zimmerman, John Korienek,
Ron Miksa, Karen Kephart, Eileen Hahn and Wanda Newby of FNAL Particle Physics Division for their outstanding work in the design and construction of the detector. The authors acknowledge the support of Gianfranco Ferioli (CERN) and Roland Jung (CERN) for conversations on OTR detectors. We would also like to thank Stephen Pordes (FNAL), Bob Webber (FNAL) and Manfred Wendt (FNAL) for their continued encouragement.

\section{REFERENCES}

[1] P. Goldsmith and J. V. Jelley, "Optical Transition Radiation from Protons Entering Metal Surfaces," Phil. Mag., Vol. 4, 836-844 (1959).

[2] C. Fischer, "Results with LHC Beam Instrumentation Prototypes," Proc. DIPAC, Grenoble, France (2001).

[3] V. E. Scarpine, A. H. Lumpkin, W. Schappert and G. R. Tassotto, "Optical Transition Radiation Imaging of Intense Proton Beams at FNAL," IEEE Trans. Nucl. Sci. 51, 1529-1532 (2004).

[4] V. E. Scarpine, A. H. Lumpkin and G. R. Tassotto, "Initial OTR Measurements of $150 \mathrm{GeV}$ Protons in the Tevatron at FNAL," Beam Instrumentation Workshop, May 2006, AIP Conf. Proc 868, p. 473.

[5] G. R. Tassotto, V. E. Scarpine, A. H. Lumpkin and R. M. Thurman-Keup , "Optical Transition Radiation Imaging of $120 \mathrm{GeV}$ Protons Used for Antiproton Production at FNAL," presented at the 2006 IEEE Nucl. Sci. Symp., San Diego, CA.

[6] J. Carbone, J. Zarnowski, M. Pace, S. Czebiniak and R. Carta, "Megarad and Scientific CIDs," Proceedings of SPIE, 2654, 131-138 (1996).

[7] W. J. Smith, Modern Optical Engineering, $2^{\text {nd }}$ ed. (Boston, MA: McGraw-Hill, 1990, p. 52).

[8] V. E. Scarpine, C. Lindenmeyer, A. H. Lumpkin and G. R. Tassotto, "Development of an Optical Transition Radiation Detector for Profile Monitoring of Antiproton and Proton Beams at FNAL," Proc. of Particle Accelerator Conference, Knoxville, TN, 2381-2383 (2005).

[9] S. Kopp, "The NuMI Neutrino Beam at Fermilab," Proc. of Particle Accelerator Conference, Knoxville, TN, 1-3 (2005).

[10]S. Kopp, et al., "Segmented Foil SEM Grids at Fermilab," Proc. of Particle Accelerator Conference, Knoxville, TN, 2821-2823 (2005).

[11] A. H. Lumpkin, V. E. Scarpine and G. R. Tassotto, "Initial Far-Field OTR Images Generated by 120GeV Protons at FNAL," Proc. of Particle Accelerator Conference, Albuquerque, NM 2007. 